\title{
Ecological and Operational Aspects of Continuous Descent Approach - Croatian Case
}

\author{
Doris Novak, Tomislav Radisic, and Mira Pavlinovic \\ Faculty of Traffic and Transport Sciences, Department of Aeronautics, Zagreb, Croatia \\ Email: \{dnovak, tradisic, mira.pavlinovic $\} @$ fpz.hr
}

\begin{abstract}
The impact of air traffic on total air pollution is a measurable value. This paper will present, on the Croatian example, the benefits of Continuous Descent Approach procedure in terms of reducing atmospheric emissions by reducing fuel consumption. Air traffic is accountable for $2 \%$ of global $\mathrm{CO}^{2}$ emissions. Although that is a small percentage, due to the higher altitudes at which the emissions are released, total impact on the global warming is considered to be between $3.5 \%$ and $5 \%$. Urgent action is required to reduce fuel burn and to make emissions savings, in order to reduce operating costs of airlines (financial aspect), and to reduce release of greenhouse gas emissions (environmental aspect). Also, it is imperative to reduce aviation noise impact on public in general. Introducing Continuous Descent Approach, as a flight operating technique in commercial aircraft operations will be a mean to achieve ecological and financial objectives. The presented results and the methodology can be used in process of operational procedures design for approach phase of the flight.
\end{abstract}

Index Terms - continuous descent approach, aircraft trajectory, air traffic.

\section{INTRODUCTION}

The impact of aviation on climate changes is primarily manifested in the burning of fossil fuel by the aircraft's engines during cruise phase of flight at altitudes in the upper troposphere. Modern commercial jet aircraft use less fuel per mile flown at higher altitudes. Therefore, the goal of every airline is to fly their aircraft at high altitudes for the most of the flight. In this way the fuel consumption is decreased, which is beneficial for the company bottom line, and greenhouse gases emissions are reduced which is beneficial for the environment. Also, noise at the ground level is lower when the aircraft flies higher.

In ideal case the aircraft will remain at high cruising altitude until it reaches vicinity of the airport and when it should descend continuously to the ground without using engine power. Due to other air traffic and aircraft limitations this is not always possible, so the aircraft needs to level off once or twice during the descent phase of flight. Levelling off at lower altitudes burns additional fuel and consequently increases greenhouse gases emission and noise at the ground level. It is possible, however, to restructure traffic flows around the airport

Manuscript received January 2, 2014; revised March 27, 2014. and introduce aircraft operating guidelines that will enable significant reduction in distance flown at lower altitudes. These aircraft operating procedures or techniques are commonly called Continuous Descent Approach/Operations (CDA/CDO). Continuous Descent Approach is an aircraft operating technique in which an arriving aircraft descends from an optimal position with minimum thrust and avoids level flight to the extent permitted by the safe operation of the aircraft and compliance with published procedures and ATC instructions [1]. In ICAO Document 9931 the generic term Continuous Descent Operations (CDO) has been adopted to embrace the different techniques being applied to maximize operational efficiency while still addressing local airspace requirements and constrains. The focus of efforts in Europe is to detect solutions and conduct operational actions that will lead to fuel and emissions savings in the short term. That is also a general objective of the Flight Efficiency Plan [2] which in five points ensures implementation of operational actions. One of the action points is efficient TMA design and utilization, through implementing CDA. It is expected that implementation of proposed Plan will bring benefits at approximately 470,000 tons of total fuel burned per year, and 1655,000 tons of $\mathrm{CO}_{2}$ per year.

The development and implementation of Continuous Descent Approach procedures is announced in Croatia Local Single Sky Implementation Plan (LSSIP), which is a part of European Single Sky Implementation Plan (ESSIP). The ESSIP is based on a common agreed view of what needs to be changed in European ATM by all those involved in European aviation in order to achieve performance benefits in safety, capacity, cost efficiency and environment. The ESSIP report is produced each year, based on the LSSIP, to address the progress made in the implementation actions during the previous year [3]. All main aviation stakeholders such as airspace users, airport operators, regulators, other international organisations, supply industry, and European Commission are involved. ESSIP's active objectives are further described in LSSIP. LSSIP ensures the correct planning, reporting and monitoring of the common agreed implementation actions in the ESSIP on the local level. One of the agreed objectives of Croatian LSSIP [4] is implementation of Continuous Descent Approach techniques for environmental improvements at airports Zagreb and Split by the end of 2013, but up to the moment of writing this paper there is no active plan. 
One of the objectives of implementation of CDA is monitoring and reduction of environmental pollution. This will be achieved by reducing noise impact on the ground, reducing fuel consumption and atmospheric emissions. With respect to impacts in the locality of an airport, aircraft noise disturbance is probably the single most important issue affecting the operation and development of airports around the world [5]. The use of CDA procedure will make a contribution to reducing noise levels during approach phase in the vicinity of Zagreb and Split airport up to 5dBA. Furthermore, reduction of fuel consumption leads to reduction of greenhouse gases emission. Preliminary study carried out at the Faculty of Transport and Traffic Sciences [6] showed reduction of fuel burned by up to $150 \mathrm{~kg}$ per approach, and consequently reduction of $\mathrm{CO}_{2}$ emission in atmosphere by up to $450 \mathrm{~kg}$ per approach. For Croatian national airliner Croatia Airlines just on Airbus A319/320 fleet during approach at Zagreb airport this means up to 7,000 tonnes of $\mathrm{CO}_{2}$ emissions less in the atmosphere per year.

On a broader scale, noise and gas emissions have a societal or external cost which burdens the economy. The adverse effect of excessive noise from all sources are estimated to cost between 13 and 38 billion per annum in the EU States alone, and aviation may account for up to $5 \%$ of this figure. Use of fossil fuel is fundamental environmental issue both from a finite global resource and from an emission perspective. If only 10 litres of fuel could be saved for each flight in European airspace, this would equate to 80,000 tons saved per annum [2]. Reduction in fuel consumption directly decreases aircraft operating costs. By implementing CDA procedures it is expected that the fuel consumption for the national airliner Croatia Airlines will decrease by 5\% per approach for landing. Stakeholders that will benefit the most are the ones that use Zagreb and Split airport for majority of their flights; in this case, national and other airlines. It is expected that the economical benefits for Croatia Airlines in terms of fuel and flight-time savings when applying the CDA technique will be up to 1,2 million USD per annum. Reduced cost per approach and landing at Zagreb and Split airports will bring those airports on par with regional airports already using CDA procedure. This will make Zagreb and Split airports more competitive and therefore more profitable

\section{SimUlation OF CDA TRAJECTORIES}

Simulation of different descent profiles were made to elaborate reduction of greenhouse gas emission by implementing CDA technique during the approach phase of flight. The simulation was based on aircraft trajectory predictions. There are several different options to model aircraft movement, some of which are look-up tables, kinetic and kinematic models. Kinematic models, such as EUROCONTROL's Base of Aircraft Data (BADA), are based on the fact that commercial passenger aircraft are operated at relatively small flight path angles. It is an assumption which allows significant simplification of complex differential equations that govern the aircraft motion. The aircraft model behind BADA is referred to as the Total Energy Model (TEM), which can be considered as being a reduced point-mass model. Fig. 1 shows model architecture used for the purpose of simulation of CDA trajectories. A three dimensional point-mass model for a standard civil aircraft was used to emulate aircraft dynamics, while the aircraft operating mode was characterised through a set of discrete variables [7].

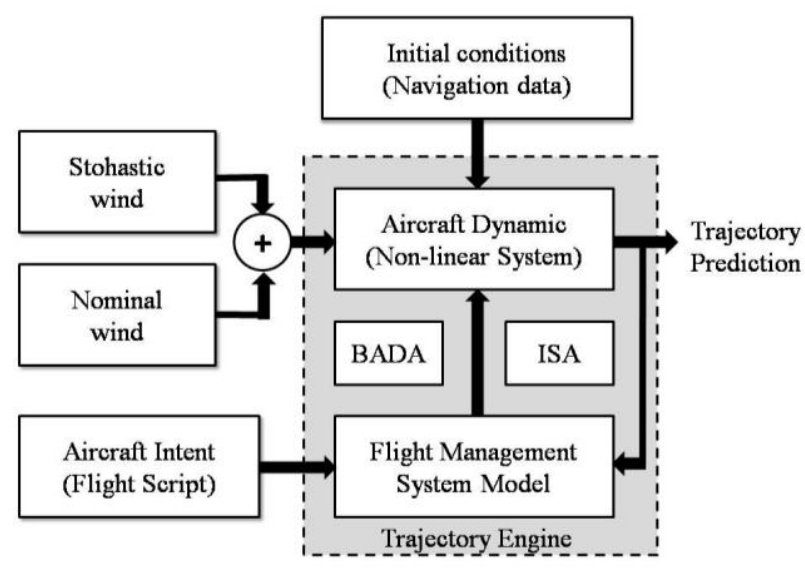

Figure 1. Model architecture for the trajectory prediction [7].

There are two main systems within the trajectory prediction engine - Aircraft Dynamic System and Flight Management System. They are in constant interaction, and in presented form, trajectory prediction model is very similar to the autopilot modelling. Aircraft Dynamic System determines the change of aircraft state variables, and is based on six state variables, four inputs, and three disturbances. Output of this system effectively provides trajectory prediction, since three of the six state variables represent aircraft coordinates. The second is the Flight Management System model which, based on aircraft present position, flight script, operational procedures and limitations, and a number of other factors, determinates the change in aircraft inputs which ensures fulfilment of flight script goals. Aircraft performance and atmospheric conditions influence to both systems, so they are integral part of prediction engine, as same as BADA and ISA database. Also, with defined flight scenarios (mission parameters), other information is needed for trajectory predictions, such as wind data.

For the purpose of simulation modelling, several flight scenarios were defined based on Airbus A319 aircraft flight performances. For simulation purpose, the ideal approach started at the maximum flight altitude which is equal to the aircraft ceiling - 39000ft. Also, from that altitude aircraft begins with continuous descent at the constant airspeed without or minimum engine power (idle thrust). This basic trajectory was compared to each individual case of 'step down' approach trajectories, which were designed by adding the extra horizontal segments to the flight path on different altitudes. One horizontal segment was assigned to one flight profile and was 1000 meters lower than the previous one. The model used total of 10 aircraft trajectories with different altitudes of the horizontal segments, starting from aircraft 
ceiling altitude - 12000 meters, and ending with lowest altitude of 2887 meters. Each horizontal segment in different trajectories had the same length of $50 \mathrm{~km}$.

The simulation also considered different aircraft airspeeds and masses during the approach phase of flight. Different airspeeds were considered at intervals of $25 \mathrm{kts}$, starting from $150 \mathrm{kts}$ and ending with $300 \mathrm{kts}$. Although this range of speed during the approach is unrealistic from operational point of view, the research objective was to demonstrate correlation of aircraft performance with the proposed CDA procedure over the wide range of technically possible conditions. The impact of aircraft mass was also considered with aircraft masses in range from 50 to 70 tones. For that purpose, a total of 210 different flight trajectories were considered, to cover the whole spectrum of possible variations within defined parameters.

\section{THE RESULTS}

Each of simulated flight trajectory confirmed that the fuel consumption was lower when the aircraft maintained higher altitude. The ideal condition for minimum consumption in the model was with aircraft mass of 50 tons at cruising speed of $225 \mathrm{kts}$ up to the top-of-descent point (point at cruising altitude from which descent begins). From top-of-descent (TOD) point, aircraft started continuous descent phase of flight without any horizontal segment. For that particular (optimal) case, the total fuel consumed was $113 \mathrm{~kg}$. By increasing aircraft mass, the optimal cruising speed also increased (for aircraft mass of 70 tons it was $250 \mathrm{kts}$ ), and fuel consumption also increased by $22 \%$ for an optimal case.

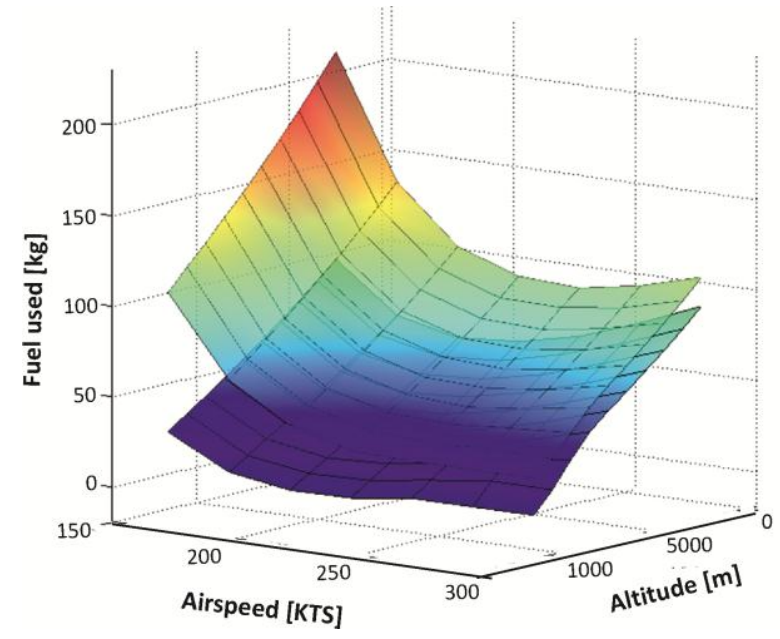

Figure 2. Function of altitude, speed and fuel consumption for simulated scenarios.

By reducing the altitude of horizontal segment in approach trajectory, the flight efficiency was also established. For flight trajectory of $50 \mathrm{t}$ aircraft with the horizontal segment at 8000 meters, the fuel consumption was $24 \%$ higher than in the optimal case $(140 \mathrm{~kg})$. For the approach trajectory with horizontal segment at the lowest altitude $(2887 \mathrm{~m})$, the fuel consumption was $177 \mathrm{~kg}$, which is $56 \%$ higher than optimal. During the approach with horizontal segment at $8000 \mathrm{~m}$ and aircraft mass of
$70 \mathrm{t}$, fuel consumption was higher by $22 \%$, and with horizontal segment at $2887 \mathrm{~m}$ it was $54 \%$ higher than optimal for that particular aircraft mass. Fig. 2 shows functions of horizontal segment altitude, aircraft speed, and fuel consumption as three-dimensional areas. It should be noted that the fuel consumption is represented by values of increased consumption from optimal case. The lower area represents fuel consumption for $50 \mathrm{t}$, and higher for $70 \mathrm{t}$ aircraft. It is also shown that the combination of higher mass with lower airspeeds represents negative factor for fuel consumption parameter.

Considering the typical descent profile for $50 \mathrm{t}$ aircraft, it was shown that $200 \mathrm{~kg}$ of $\mathrm{CO}_{2}$ will be emitted into the atmosphere during the horizontal segment at $3000 \mathrm{~m}$. In case of Zagreb airport which have around 7000 approach operations of A319 for runway 05 per year, that makes total of 1400 additional tons of $\mathrm{CO}_{2}$ into the atmosphere. This can be significantly reduced by shortening or completely removing horizontal flight segment during descent/approach phase of flight.

\section{ECOLOGICAL IMPACTS OF CDA}

Presented results are for ideal cases. In real operational air traffic situations, it is clear that it will not be possible to implement such procedure form TOD, so the benefits in terms of reducing $\mathrm{CO}_{2}$ emissions will be slightly lower. Carbone dioxide is a naturally occurring gas, but also a by-product of burning fossil fuels. Besides to $\mathrm{CO}_{2}$, byproduct are also the reactive nitrogen oxides ( $\mathrm{NO} x-$ the sum of $\mathrm{NO}$ and $\mathrm{NO}_{2}$ ). They are also released into the atmosphere. In contact with water vapor, which is also significantly present in engine exhausts, it creates a nitric acid, which is responsible for acid rains which affect concrete structures and environment in global sense. Engine that was used in this simulation is a typical representative of the engine used in the medium range aircraft. This engine produces 10,32 g NOx for each kilogram of fuel used, during approach power settings [8]. This means that during typical descent of 50 tons aircraft, it produces 2064 grams of NOx. In contact with water vapor this creates 2830 grams of nitric acid. Therefore, on an annual basis in case of 5000 landings/approaches at only one airport, $14150 \mathrm{~kg}$ of nitric acid is released through participation in the atmosphere. Fuel combustion in aircraft engines or incomplete combustion releases a significant amount of hydrocarbons, carbon monoxide and soot. Hydrocarbons are considered environmental pollutants and carbon monoxide in the presence of NOx and volatile organic compounds form a photochemical smog that has numerous negative effects on human health. Apart from the exhaust gas emissions, aircraft noise also pollutes the environment. Noise is considered all unwanted sounds that interfere with human life and work, and it reduces quality of life. During aircraft operations, the most exposed to noise is human population in the vicinity of airports. Aircraft in flight mainly produces noise by flying through the air (aerodynamic noise) and by engines. Aerodynamic noise is a result of local air pressure differences that are reflected as noise. Therefore, as the aircraft has better aerodynamic shape, the level of 
noise that it generates is lower. Air vortexes, which are caused by turbulent airflow, increase the intensity of noise. During approach phase of flight, extended landing gear and flaps influence aircraft aerodynamics and create such turbulent flow. The noise generated by the engines is directly related to the operating mode. The greater the thrust produced by the engines, the greater the noise. Accordingly, the aircraft in level flight and during the climb phase when more thrust is required generates more noise in contrast to the approach phase when power is usually minimal. During the CDA procedure, aircraft avoids the horizontal segment of flight and engine power is at the minimum during entire descent phase. This reduces the noise level during the approach/descent phase of flight. In the open space, where there is no sound reflection, the noise level is inversely proportional to the distance from the noise source. For example, an aircraft flying at $12,000 \mathrm{ft}$ is heard 16 times weaker than an aircraft flying at $3000 \mathrm{ft}$. CDA requires that aircraft maintain cruising altitude for as long as practicable, and once the descent starts, to fly without any adding additional thrust until intercepting the ILS. Thus, the advantage of the CDA in terms of noise is two-fold - the noise level is lower for the sake of flight at higher altitude over a longer period of time, and engine noise is reduced as it operates on lower thrust all the time during the descent and approach.

\section{RNAV TRANSITIONS AND CDA}

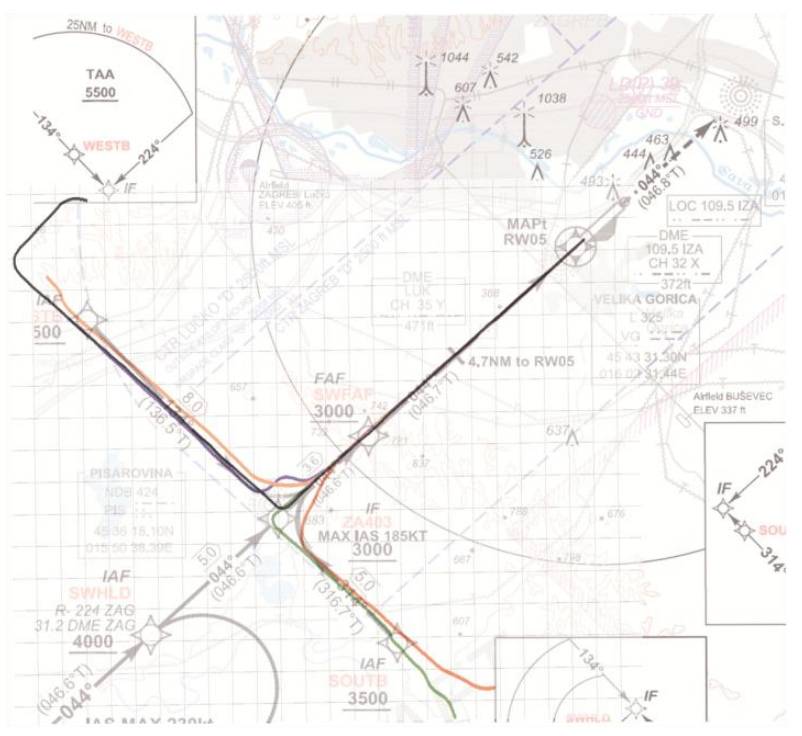

Figure 3. Tracks of RNAV procedure for Zagreb airport

Evaluation of applying CDA should be performed in terminal airspace on RNAV transitions. An RNAV (Area Navigation) transition is the instrument arrival procedure which defines the flight path of the aircraft according to predetermined waypoints. The position of the waypoint is determined by GNSS or DME/DME, according to the area navigation concept and performance based navigation specification used. In order to obtain more accurate measurements and results of fuel consumption (which is directly related to the gas emissions), it is necessary that the aircraft remains as long as possible on prescribed track, with minimum radar vectoring by air traffic control. De Muynck, et al. [9] evaluated a set of enablers to improve the CDA operations in terminal airspace of arriving traffic from TOD to touchdown at the runway. Different scenarios are simulated under different conditions, and the results confirm that the CDA is a promising procedure in terms of noise abatement and environmental improvement near airports. Moreover, it is needed to design RNAV procedures for major airports in Croatia to allow track keeping and CDA techniques accordingly. For Zagreb airport, RNAV procedure was designed and tested for flyability (Fig. 3).

The objective of the flight trails conducted in September 2013 was to evaluate the instrument flight procedure and to assure that the navigation source (in this example GNSS was used) supports the procedure. Also, obstacle clearance, and checks of flyability of the design were made (complexity of design, evaluation of cockpit workload, and check for correctness of information). Due to a fact that the research results confirmed that CDA can be applied in realistic traffic conditions [9], it is necessary to further investigate interoperability between air and ground systems, aircraft avionic systems and functions which helps to plan and conduct the CDA and ground system that supports CDA operations at high density airspace. Also, it is necessary to adjust the procedure and technique to aircraft types and airspace.

\section{CONCLUSION}

The impact of air traffic on air pollution in total is a measurable value. The worldwide efforts are made to reduce that impact to an acceptable level. The results presented in this paper confirmed correlation between the fuel consumption and aircraft descent profile/altitude. There are also other parameters that can influence final results, such as operational constrains, airspace complexity and air traffic controller workload. Nevertheless, the trajectory prediction model and presented results can be of significant help in designing CDA (and other) air navigation procedures, and airspace operational environment. At the first look it may seem that the figure of around $50 \mathrm{~kg}$ of fuel saved during the approach is not of particular interest to the aircraft operator, but multiplying it by the number of flight operations in defined time period can be of significant economical meaning. Also, knowing that an approach procedure on an airport is designed to reduce fuel and gas emissions can positively influence the quality of life of people in community around the airport and beyond.

In normal operations, there is no possibility to perform CDA from TOD to final approach fix (point), or to touchdown, due to airspace congestion and other operational limitations. Therefore, the procedure should be implemented gradually, starting with night operations, and when the traffic situation allows. It is a gradual process in most countries, but it is important to establish some kind of culture in performing such operations. The long-term benefits of CDA procedure application are reduction of noise impact on the ground, reduction of fuel consumption and reduction of atmospheric emission. It is 
expected that airliners will continue to perform this procedure while it saves flight-time and fuel and consequently leads to cutting costs. In Croatia, Zagreb and Split airports will be more competitive on the market and appealing to international airliners already performing CDA procedure at other airports. In the longrun general population will be less exposed to noise and emission of atmospheric gasses.

\section{ACKNOWLEDGMENT}

This work was supported by a grant Kratkorocna financijska potpora istrazivanju (2013-ZUID-21) from the University of Zagreb, Croatia.

\section{REFERENCES}

[1] Continuous Descent - A guidance to implementing Continuous Descent, EUROCONTROL, October 2011.

[2] Flight Efficiency Plan - Fuel and Emissions Savings, IATA, EUROCONTROL/CANSO, August 2008.

[3] The ESSIP Report for 2010, EUROCONTROL.

[4] The LSSIP Croatia Year 2012 - Level 1, EUROCONTROL 2013.

[5] P. Upham, et al., "Environmental capacity and airport operations: current issues and future prospects," Journal of Air Transport Management, pp. 145-151, 2003.

[6] D. Novak, T. Bucak, and T. Radišić, "Development, design, and flight test evolution of CDA procedure in FIR Zagreb," Promet Traffic and Transportation, Zagreb, 2009, pp. 319-329.

[7] M. Porretta, et al., "Performance evaluation of a novel 4D trajectory prediction model for civil aircraft," Journal of Navigation, vol. 61, no. 3, pp. 393-420, July 2008

[8] ICAO Aircraft Engine Emissions Databank. [Online]. Available: http://easa.europa.eu/environment/edb/aircraft-engineemissions.php

[9] R. J. de Muynck, et al., "Enabling technology evaluation for efficient continuous descent approaches," in Proc. 26th International Congress of the Aeronautical Sciences, 2008. 